\title{
Organizational Commitments to Gender Equality Programming: Resistance, Externalizing, and Opportunities for Gender Mainstreaming
}

\author{
Lan Thi Nguyen
}

\section{Introduction}

Gender inequality is one of the most significant human rights challenges of our time (UN Women: $n$.d.). Women are disproportionately affected by higher poverty rates, gender-based violence, child marriage, and early mortality with adolescent births. Building on previous international commitments designed to put women's rights and gender equality as a cross-cutting theme in international development, the Beijing Platform for Action (arising out of the 1995 Beijing Conference) prioritized several areas of action needed to promote women's rights and gender equality. Among these priorities, the Platform highlighted the issue of gender mainstreaming strategies and gender equality programming. These priorities have increasingly been adopted by non-governmental organizations (NGOs) and civil society organizations (CSOs) since the 1995 Beijing Conference.

Before the conference, other international commitments also called for more significant investments in women's rights, such as the Convention on the Elimination of Discrimination against Women (CEDAW) - an international treaty adopted in 1979 by the United Nations General Assembly ratified by 189 countries. More recent commitments to gender equality can be found in the 2000 Millennium Development Goals (MDGs, particularly Goal 3: To Promote Gender Equality and Empower Women) and the updated international priorities under the Sustainable Development Goals (SDGs, particularly Goal 5: Gender Equality) which was adopted by 193 countries. All ten countries examined in this study (Malawi, Kenya, Nepal, Uganda, Senegal, Ghana, Vietnam, Peru, Guatemala, and Tanzania) have agreed to and ratified these international commitments while also committing to designing national-level policies, machinery, and services to promote women's empowerment and gender equality. As a signpost of the global solidarity needed to achieve SDG 5, one participant from Uganda noted: "almost all countries have international standards regarding gender equality... We are all striving for the same goal". Development NGOs have demonstrated mixed progress on implementing international priorities to address gender inequality and implement gender mainstreaming strategies (Tiessen 2007).

This paper examines this 'mixed progress' and begins by defining gender mainstreaming and summarizing the contribution of gender mainstreaming to 
development. Next, an overview of the relevant literature highlights the problems and possibilities for improved (or transformative) gender mainstreaming, and then considers how development NGOs in the Global South have navigated gender equality priorities concerning resistance, externalizing, and integration of gender mainstreaming with support from international development volunteers (IDVs). The paper then turns to explore the diverse experiences of development NGOs concerning gender mainstreaming priorities, with attention to resistance elements to adopting gender equality and women's empowerment (GEWE) programming. Findings are buttressed by examples of opportunities for advancing GEWE through innovative programming within the partner organizations that support and guide IDVs. Finally, the paper analyzes activities that externalize (project gender inequality issues onto rural communities, rather than internalizing gender equality priorities within the development organizations) GEWE priorities to activities in rural or remote communities and conclude with implications for practice.

\section{Literature Review}

GEWE has a long history dating back to international commitments and conventions. The record includes the 1979 Convention on the Elimination of all Forms of Discrimination against Women (CEDAW), the Beijing Platform for Action (1995), and more recently, the MDGs and SDGs. The Beijing Platform for Action, in particular, outlined the need for a gender mainstreaming approach to ensure that "gender perspectives and attention to the goal of gender equality are central to all activities - policy development, research, advocacy/dialogue, legislation, resource allocation, and planning, implementation and monitoring of programmes and projects" (UN Women: n.d.).

The Beijing Platform for Action prioritized gender mainstreaming as the mechanism to achieve gender equality. Two critical aspects of different definitions of gender mainstreaming exist: (1) the institutionalization of gender concerns and experiences within the organization and (2) women's empowerment (Moser/Moser 2005). To fully integrate gender priorities requires considering GEWE in administrative, financial, human resources management, and other organizational procedures, leading to a long-term transformation in staff's attitudes, behaviors, cultures, and operations (Tiessen 2007). Gender empowerment means promoting women's participation in decision-making processes, making their voices heard, recognizing that women have agency, ability, and capacity to make choices and make decisions related to their own lives (Moser/Moser 2005).

Despite clear definitions and national commitments to gender mainstreaming, development organizations have been slow to adopt gender-sensitive programs and priorities. Some of the reasons attributed to the slow uptake of gender 
mainstreaming are lack of staff capacity and training in gender equality, an organizational culture that prevents GEWE, and negative attitudes among staff toward women or gender priorities (Derbyshire 2002; Moser/Moser 2005). However, effective gender mainstreaming practice within development organizations requires a broad range of commitments, from human resource policies such as anti-discrimination policies, budget allocations to integrate gender equality across diverse program priorities, staff training opportunities, coordinated efforts across the organization with support from gender-trained experts, and operational practices that mainstream gender equality into programs, projects, and activities (Jahan 1995). Examples of operational gender mainstreaming practices include increasing focus on the gender-related impacts in project design implementation and evaluation of projects and programs, sex-disaggregated data collection, and gender analysis to ensure that projects/programs can challenge unequal power relations.

The success of gender mainstreaming activities relies heavily on all members' internal responsibility and commitments within organizations, including all staff. It also demands careful attention to masculinist or patriarchal organizational cultures that perpetuate negative attitudes, working environments, and practices that discriminate against women staff and community beneficiaries (Valk 2000; Rao/ Kelleher 2016). Examples of exclusionary practices include organizational practices that may not reflect the needs of those who are responsible for childcare (Wallace 1998). There are also examples of forms of resistance and "backlash" to gender equality and gender mainstreaming, such as denial of the issue of gender inequality and lack of awareness of gender issues (Flood et al. 2020).

Even when efforts are in place for mainstreaming gender within development organizations, bureaucratic norms and practices may prevent gender equality priorities from translating from policy goals to bureaucratic performance and program delivery (Tiessen 2019). Moreover, bureaucratic norms and practices may result in neoliberal and instrumentalist techniques of governance (Eerdewijk/ Davids 2014), resulting in checking boxes of gender indicators to meet a requirement rather than meaningful change to policy and practice. Thus, achieving a 'transformative' vision of gender mainstreaming requires institutional change and addressing the masculinist status quo within institutions and development organizations (Parpart 2014). This framing explores how partner organizations navigate gender mainstreaming with support from international development volunteers in the Global South.

\section{Methods}

Interviews were conducted in 2018 - 2019 with 150 partner organization staff in ten countries. Once all interviews were transcribed, data were coded to identify common themes throughout data analysis. Finally, data were analyzed using 
discourse analysis. The introduction to this special edition has more detailed information on the methodology for data collection and the study of findings.

\section{Findings}

Three significant findings frame the analysis of the diverse experiences of these staff members, including (1) challenges and resistance to gender mainstreaming; (2) externalizing priorities for transformative change in gender relations as a problem occurring "somewhere else" in rural and remote areas, and (3) opportunities for improved gender awareness in transnational spaces.

\subsection{Challenges and Resistance to Gender Mainstreaming in Partner Organizations}

Some of the partner organization staff acknowledged that gender is not the main focus of their work. Therefore, they do not have many activities related to promoting gender equality and gender mainstreaming. Feedback received from a Vietnam-based partner organization staff demonstrated rationales provided by some development organizations for why gender was not perceived as an essential lens for their work. For example, for one organization with a "focus on hospitality management, restaurant management," gender equality is not considered an important area of focus since their activities are seen as gender-neutral. Likewise, another participant from Vietnam noted: "There is no difference between men and women, male and female, there is no program focusing on gender, as we respect 'diversity' suggesting that all students are treated fairly and equally, even in organizations where fewer than 10 percent of their participants involved in information technology training are women. The main area of focus was to increase overall enrollments with little consideration for the lack of interest in their programs from diverse groups.

Other participants considered gender equality programming as not their priority in terms of organizational work. As a participant from Guatemala noted, "the culture of the superiority of men is talked about sometimes. But we don't talk about these things in the organization [or] in our offices. This is not part of what we do". The participant explained that respect for women and commitments to gender equality were highly valued within the organization, but these commitments were not linked to organization commitments, policies, or actions.

Several participants from different countries referred to perceive success at the national level in achieving gender equality. For example, in Vietnam and Nepal, several participants noted that gender equality had been achieved already in the country, citing education enrollments of boys and girls and legal mechanisms in 
those countries, as a participant from Nepal noted, "both men and women are equal in our law, and we also treat them equally in our school."

These findings highlight a perception among some partner organization staff that gender mainstreaming is not a priority at the organizational level, and little if any time is devoted to addressing organizational practices that might contribute to program design biases or inequality in the treatment of certain staff members. For organizations that do not see their programs as specific to gender equality projects, gender issues were considered irrelevant and the focus of other organizations. Some of the limited contributions to gender mainstreaming in their organizations were explained as a lack of capacity to do this type of work. Interviewees noted that they faced challenges in carrying out gender mainstreaming due to their limited understanding of gender issues and their lack of expertise and confidence to work on issues of gender equality. One interview participant from Vietnam noted that their organization lacked the capacity and expertise to work on gender issues, so they relied on partner organizations to bring in that expertise. Partnering with UN Women allowed one organization to design better its training programs with gender equality priorities in mind.

Several organizations also mentioned the need for gender training, noting that donor or partner organizations in the Global North often provided some level of training. However, due to limited time, financial, and human resources, gender training and capacity building for staff have been limited. In addition, leadership in partner organizations has not made gender training a priority.

During several interviews, when asked if the organization has strategies to address GEWE, organizational staff referred to data about the percentage of women who had participated in events. In several countries, partner organizations referred to their reporting guidelines and commitment to recording women and men as beneficiaries of training or project activities.

However, these strategies of recording sex-disaggregated data did not often include strategies to understand why the inclusion of women or diverse groups was necessary, nor did they consider the nature of their participation, nor the impacts of the activities in relation to gender issues. In each project report for Canadian funders, partner organizations are required to provide information about how their programs have addressed gender issues. However, when being asked about how they completed such reports, several partner organizations highlighted how they included only the number of women who attended activities.

Addressing the structural inequality and social norms and beliefs that perpetuate gender inequality were generally not discussed due to limited awareness and understanding of broader gender issues and the tendency among partner organizations to focus on individuals rather than societal realities. For example, organization 
staff talked about the importance of ensuring gender balance in the number of participants. Furthermore, annual reports recorded the number of male and female participants and, in some cases, information about salaries for men and women. A respondent from Vietnam noted: "the number of the female teachers is the same as male teachers, and the rate salary is the same... That is the way we deal with gender."

Interviewees sometimes demonstrated resistance or refusal to consider gender issues in their work. The resistance was often linked to narrow perceptions of what constitutes equality. For example, in Vietnam, a participant noted: "There is no difference in the salaries of men and women, women might get higher when they have higher position and experience, [or demonstrated] effectiveness." In other reflections on gender equality, participants shared a limited understanding of gender inequality by linking success to the strength and hard work of the individuals rather than to supportive environments. For example, as one participant noted, "in Vietnam,... female members are not weak. Female lecturers and students undertake many important and hard responsibilities." These examples did not include consideration of the gender division of labor that can take place within workplaces (additional labor women may need to complete as part of their service roles) or the excessive workload they may experience in their homes (taking on more responsibilities caring for children and other family members). Despite these views of success being linked to hard work, some of these same respondents did observe that women lack voice or decision-making power, particularly in some of the highest positions of the organizations where women are less likely to be represented. While gender inequality was observed within the highest positions within the organization, some participants viewed this as generational and changing. As a participant from Vietnam explained: "Our next generation of our younger sisters/ our daughters will be different." The participant considered these changes emerging as young women in the country had different views about their relationships to their husbands and their demand for greater equality. While this was viewed as the vision for the future, "in reality in Vietnam now ... there is still gender inequality even in [the] office".

Other examples of resistance to improving or increasing organizational commitments to gender equality pointed to observations that women are treated better than men or that women and girls are given preferential treatment. In Ghana, some participants referred to programs that provide assistance to women and girls and thereby leave men behind. Participants expressed resistance to a greater focus on gender equality because they perceived too much attention focussed on women and girls already.

Other examples of perceived preferential treatment for women were noted in relation to the "two celebration days for women: one for Vietnam [Day] and one for 
International Women's Day. It means that we care enough for everyone... [and that, in fact, the woman] dominates the family". The reference to these two days of celebration of women was seen as a commitment off respect for women. The perception of equal treatment between men and women also translated into perceptions of the equal treatment of girls and boys in the country. As one participant noted, with family sizes being smaller, "the boys and girls are equally treated." Importantly, both boys and girls were sent to school to get a better education in this community. However, a preference for sons was seen by interviewees as a possibility for families who already have a daughter. Otherwise, both boys and girls were generally believed to be treated equally.

In Uganda, participant organization staff referred to new conflicts arising between men and women in the workplace. The concern was that gender equality work, and changes were creating what were perceived to be new inequalities: "I'm seeing a generation where women are more empowered than men, and then we would need to go back to fighting for the rights of men." Another participant from Uganda expressed similar concerns about the "negative consequences" of gender equality work whereby "people feel that maybe women are being promoted, and not the male counterparts." A participant from Kenya expressed concerns that gender equality programming was shifting the balance in favor of women noting that a lot of work is being done for a girl child and "now boys are becoming vulnerable." This change was considered a cause for concern as it could lead to other forms of inequality and bring Kenyan communities "back to where we came from." This growing imbalance was noted by yet an additional participant in Uganda who said: "I'm seeing a generation where women are more empowered than men, and then we need to go back to fighting for the rights of men." The participant concluded that they should focus on equality rather than women's empowerment and avoid being "extremist."

Many of the examples of resistance to discussions of gender equality were explained in reference to cultural practices that highlight men's and women's different roles in society. The most common forms of resistance to gender equality were noted on how the 'beneficiary' communities felt about gender equality and the low level of acceptance of concepts like gender equality in their culture. For example, in Kenya, one participant remarked how society is still: “... steeped in African traditions and is a patriarchal society where men are very dominant and ... women are trying to come to the same level through equality," which can lead to "resistance in this community" by both men and women. According to another participant in Peru, resistance to gender equality was perceived to be a challenge in the projects they implement in the communities where you encounter "machista men" as well as women community members who do not want to disrupt patriarchal norms and who may "close the door" on gender equality programs because the communities do not feel comfortable addressing societal changes to gender relations. In recognition of 
these multiple areas of resistance within communities, notions of social inclusion and gaining community buy-in were considered essential strategies for addressing gender equality. In the absence of such buy-in, participants referred to the need to tread carefully when doing gender equality work because cultural norms and practices make changes to gender relations a challenge, and gender inequality was generally considered an issue outside the organizations or externalized as elaborated in the section below.

\subsection{Perceptions of Gender Inequality as a Problem "Somewhere Else" in Rural and Remote Areas}

Perceptions of gender inequality were often directed to problems specific to "other" remote or rural areas. During interviews, many participants began by denying any gender inequality in their cities or their organizations but, throughout the discussion, turned to their perceptions of specific gender-related issues experienced by women as a result of gender inequality in the communities where programs are implemented. For example, one participant from Vietnam referred to support provided to a woman who had become a widow and therefore lost her family income and support, forcing her to commute to the city each day to sell food for survival. When probed, the interviewee began to reflect on why the widow had these discriminatory experiences and explained how unfair societal gender norms and cultural practices result in stigma and discrimination explicitly experienced by widowed women in rural areas.

Applying an intersectional lens to the interview discussions combined with a focus on women's experiences with regional location, ethnicity, age, and socio-economic differences opened up new conversations about participants' personal experiences with gender inequality. In so doing, however, the partner organization staff often framed their discussion from an 'externalizing' lens towards gender inequality as something "out there" in other communities rather than experiences that impact women in their localities.

Another interview participant talked about working in some of the poorest provinces of Vietnam and noted that "gender issues are still big issues in far remote areas." Because of this, the work involved promoting women to help them get jobs in the tourism industry. The participant further explained: "women from ethnic minorities had to work very hard, they don't have equality at all in the family, because they are not independent in the family". However, as the interviewee noted, sustainable jobs are vital to accessing income for women who have few options for work. When job opportunities in tourism are created, women can improve their lives. The interviewee also noted that these efforts are important because gender inequality is slower to be realized in the countryside, and women are not treated as equal to men. 
When revealed in the interviews, the examples of gender inequality as something 'out there' in other communities, created a starting point for further discussions about gender inequality more broadly defined. These conversations often turned to partner organization staff recognizing that the gender division of labor relates to their areas of work, with specific implications for gender relations and women's experiences of inequality, women's voice in decision making, and women's experiences of intersectional inequality. For example, discussions turned to the higher workload for women responsible for significant reproductive work and unpaid care work, leaving limited time for their career and income-earning activities, including developing a start-up business. For example, one respondent in Vietnam explained his knowledge of challenges for women in business as such: "There are some barriers for women in businesses. Many men want women to stay home and spend more time with the family, taking care of children, men and family members do not support women with business." Likewise, another female interviewee noted that "in my family, I also have a job with eight hours in office like my husband, but I still have to spend more time to take care of children and unnamed work."

In Tanzania, one participant highlighted what he saw as challenges to doing gender equality work with beneficiary communities since, as he described, an understanding of gender relations is different in the rural communities: "First of all, they [volunteers from the Global North] have to understand the Tanzanian context. I am sure where they come from, and Tanzania is two different things: you may find the issue of gender is quite different". This participant suggested requiring development organization staff "to sit with indigenous people and learn about our gender" before designing programming that may not otherwise reflect their cultural realities.

Other examples of partner organization staff reflecting on the nature of gender inequality in rural or remote areas could be found in comments provided by a Peruvian partner organization staff who mentioned the strategy required to design programs that address gender inequality when working in rural areas. This staff member was concerned because "you run the risk, as an NGO [that] wants to empower women, and in a community where there are more men - especially machista men, than women could close the door on you." The participant's concern was that community members would refuse to engage in programming that promotes women's equality if it is perceived to transgress gender norms in those communities.

\subsection{Opportunities for Improved Gender Awareness in Transnational Spaces Through International Development Volunteering}

Changing perspectives on the significance and relevance of gender equality as a development priority (both in the communities where organizations are working 
and within the organizations) have been facilitated, in part, by interactions with IDVs. Generally, IDVs were seen as bringing critical capacity-building skills in the area of gender equality programming. For example, according to staff in a Vietnamese partner organization, gender-related support from IDVs was needed because: "We are still struggling ourselves .... We don't have a gender training strategy or approach in a systematic way".

In support of the work of partner organizations, IDVs were also able to strengthen the day-to-day running of business operations through their communication and information technology skills, allowing for the intensification of host partner organizations' efforts towards GEWE. As a participant from Ghana noted:

Most of our international volunteers supported us to use social media to highlight the issue of women in our work. So there [have] been instances where we used that as a model and the involvement of the international volunteers and their expertise in ICT [information communication technology] helped us to improve our work.

At the organizational level, IDVs were valued for their fresh perspectives. As a participant from Tanzania said:

What I value the most is [their] experience and perception of things. People are coming from various environments, which is a very good thing for us. We need very fresh eyes on some old stuff so that we can modify or do them better. They say, if you are doing something for a very long time, you might develop, or you can grow monotony. Fresh eyes help us develop.

As transnational actors, IDVs also brought new perspectives and created new opportunities that were needed to change attitudes. For example, in Vietnam, volunteers brought about different perspectives to address the problem of gender inequality from various angles: "Having someone from outside the region with different perspectives, especially on gender, gave the participants a different way of understanding the issues at the global scale.”

Other partner organization staff talked about improved work in the area of gender equality as a result of partnering with IDVs. For example, as a participant from Ghana noted, the IDVs reinforced the value and significance of a gender focus in working with small-holder farmers and helped include more women in these projects. The IDVs were also instrumental in enhancing the skills and competencies of organization staff through their contributions to training. For example, in Ghana, the participant noted:

The international volunteers insist we make sure that [the training] is gender-sensitive such that it won't be all male or all female. Because of that, we try to bring both sexes to make sure that responsibilities are given to people, 
not because of their gender but to ensure that everybody in the organization has a role to play.

Other contributions provided by IDVs to organizational gender mainstreaming efforts included work to create gender policies within the organization. For example, in Uganda, IDVs provided support in relation to sexual harassment policies. They shared their experiences and knowledge from their own countries to help local universities in Uganda to develop a sexual harassment policy. A Ugandan participant expressed their appreciation for the IDVs who provided support that enabled them to incorporate gender equality into their programming. In another example, IDVs in Senegal assisted with various activities involving "gender strategizing" that helped organization staff build their capacities in gender equality programming. They also helped develop legal instruments and gender training that helped facilitate capacity building among member organizations. Finally, in Nepal, the IDVs played a role in creating action plans that helped the organization "increase the number of female members in the cooperatives, to include women in the policy and decision-making level."

Many of these changes were directly attributed to the intervention of IDVs. For example, according to a staff member in Nepal, it was "only after the arrival of the ... volunteer [that] we realized the need, and importance of, GESI [Gender Equality and Social Inclusion] in [our] organization". Another participant in Malawi reinforced the distinctive contributions made by IDVs to their programs and policies: "I can proudly say that we have what we call a gender policy, [and] it is because of the initiative from the same volunteers."

In addition to these benefits, participants also identified several challenges or areas for further consideration. These suggestions were provided to help organizations get the best support possible through transnational interactions. One example of room for improvement was the level of expertise and professional experience that IDVs could bring. Most organizations required staff with gender expertise who could provide them with technical skills and advice on gender mainstreaming and women's empowerment. However, many IDVs had not been sufficiently trained on this issue. Some partner organizations were frustrated with the lack of formal training in gender equality programming among the IDVs, especially considering the organization's need for this professional support and technical skills in gender mainstreaming. As one participant from Vietnam noted: "For the volunteers coming here, I don't think they had enough gender training or specific gender skills to help us to do gender activities. It would be useful to have a gender advisor to support us". Recognizing these challenges improved training among IDVs was considered highly valuable. 
Participants noted that partner organizations could play a more prominent role in screening applicants to ensure that IDVs came to their communities with the technical skills needed to support gender mainstreaming in the community organizations. They suggested that doing so would require better communication between the volunteer sending organizations and the partner organizations. As a participant from Guatemala noted: "[...] it would be better to have more communication between us - because we never know what is going on in the process until out of nowhere, they have a volunteer for us. It would be better to have more communication."

Other areas for consideration were the increased amount of time provided by the IDVs and closer attention to gaps created in the timing of their departure from the organization. There was a lack of continuity after volunteers left because partner organizations did not have enough personnel to fill the full-time positions required for the projects started by volunteers. As a result, the two-way flow of knowledge necessary to facilitate capacity building and long-term sustainability was negatively impacted by the limited window for integration into the community. In Ghana, a respondent felt that with the complexity of GEWE:

The duration of the volunteers is short. It is barely 11 months. For me, that is not enough for the volunteers to actually understand the culture and make any serious impact when it comes to gender equality and women empowerment. Gender issues are very critical issues; you do not empower women overnight; one year is very short.

Many respondents agreed that a limited duration negatively impacted the effectiveness of IDVs' integration into the community and thus their impacts on GEWE initiatives. As a result, the two-way flow of knowledge necessary to facilitate capacity building and long-term sustainability was negatively impacted by the limited time window for IDVs' integration into the community.

Although IDVs and volunteer programs contributed to promoting GEWE in many countries, many challenges need to be tackled to ensure the success of volunteer programs and the meaningful contribution of IDVs. Respondents expressed their frustrations with expertise and professional experience on GEWE, as many volunteers lacked formal training in gender equality work. On the other hand, while many IDVs lacked professional experience in gender equality, most were viewed as flexible and willing to learn how to assist with GEWE initiatives. Increased communications with partner organizations during the recruitment process could address this gap. In summary, IDVs' level of expertise, cultural adaptability and sensitivity; time spent in the role/country; and language barriers were frequently identified as major factors that impeded GEWE outcomes. 


\section{Analysis}

Despite international and national commitments to gender mainstreaming and prioritizing gender equality as a cross-cutting theme, development organizations face several challenges and barriers to effectively operationalizing gender equality commitments. The findings documented in this paper reveal the challenges of gender mainstreaming, including resistance to gender equality priorities, despite national commitments to international gender equality priorities.

Resistance to discussions about gender inequality is not new and is an inevitable response to social change (Flood et al. 2020). These findings reinforce various forms of resistance to gender equality and gender mainstreaming articulated in previous scholarships, such as denial of the problem, refusal to recognize responsibility to address issues, inaction to implement a change, and the use of violence and harassment (Flood et al. 2020; Moser/Moser 2005). In the paper, one of the most common forms of resistance is a perceived preferential treatment accorded to women with examples of international women's day events, programs that prioritize women and girls, and assumptions about gender equality based on existing gender norms. Problematically, however, these perceived preferences were provided without consideration for patriarchal structures that could quickly negate many of these perceived advantages.

Additional examples of resistance to gender mainstreaming were observed in relation to the simplistic approach to reporting on gender equality outcomes in project reporting. Partner organizations generally employed a Women in Development (WID) approach or liberal feminist approach that counted women participants as the basis of their evidence of gender equality work. While including women in program activities is an important starting point for addressing gender inequality, it is also insufficient for tackling the structural causes of marginalization or oppression that many women experience (Cornwall 2003). For many organizations, counting the number of women participants was seen as an effective and sufficient strategy for monitoring and evaluating gender equality results and impacts. However, this simple measure ultimately tells us little about gender equality outcomes. It is insufficient to assume that "if more are counted, they'll count more" (Nelson 2015: 41).

The simple measures of counting women participants are employed at the expense of capturing fuller, thicker understandings of gender inequality which expose the structures and institutions that perpetuate inequality and masculinist societal norms. Other important questions remain, including questions about whether women participants' voices are heard, whether decisions made by women are legitimized by men in the community, and whether differences between diverse women are considered in decision-making processes. Therefore, it is also vital to examine how gender equality initiatives help to challenge gender stereotypes and the 
structural causes of inequality, the unequal relations between men and women, and the intersectional realities of diverse members of a community. Just as "development is intrinsically about power" (Radcliffe 2015: 855), so too is gender equality about tackling the inequitable power relations that perpetuate women's oppression. Gathering this richer data (beyond the mere counting of women participants) would help expose women's experiences as they navigate social and institutional manifestations of inequality (Hay 2012) and their agency as they seek to change the structures that contribute to their marginalization.

The findings also uncovered specific examples of how gender inequality is understood by organizational staff. Specifically, beyond the resistance to address gender issues within the organization (in policies, practices, and monitoring, evaluating, and reporting activities), partner organizations noted some opportunities for considering gender inequality. However, those spaces for tackling gender inequality were primarily viewed as issues considered external to the organization or a problem to be solved elsewhere in the communities or rural areas where programs are delivered. When partner organizations were asked for more information about why gender inequality is a consideration for their programmatic work, staff members often turned to an intersectional analysis, documenting the experiences of specific groups of women such as widows or women who have very little income. In so doing, partner organizations rejected essentialisms of women's experiences and highlighted intersecting realities of oppression and inequality faced by particular groups of women. These intersectional analyses are important for understanding the unique and specific issues faced by diverse members of the community, highlighting discrimination based on age, marital status, ethnicity, education levels, socio-economic status, etc. These insights lend themselves to transformative gender mainstreaming (Parpart 2014) and can be used as examples for expanding gender mainstreaming initiatives. In addition to this more nuanced understanding of intersectional gender issues, partner organizations must also consider how gender inequality is experienced in all communities and within the organizations themselves. The assumption that gender inequality is solely an issue for the "beneficiary communities" results in few opportunities for organizational staff to consider how diverse experiences of gender inequality affect their own lives and the lives of others within their organizations.

Partner organizations see IDVs as valuable transnational actors who offer insights into gender equality strategies to be applied both within the organization and in the organization's programmatic work in the communities where they work. Several examples were provided by partner organizations of how IDVs were able to introduce gender mainstreaming activities to expose gender inequality within the organization. IDVs also helped improve exposure to new ideas and introduced policies, training, and capacity building in gender equality. In so doing, IDVs were seen 
as transforming gender relations through everyday interactions, by building confidence in GEWE programming, and by modeling behavior that fosters changes in gender norms (Tiessen/Rao/Lough 2020). Broadened perspectives on GEWE began with increased intercultural exposure between IDVs and staff members. IDVs, therefore, play an essential role in shaping how partner organizations understand power dynamics, gender relations, and root causes of inequality.

Perhaps most importantly, many of the findings highlighted challenges that need to be addressed by organizations involved in sending volunteers abroad. Volunteer-sending organizations need to pay careful attention to strengthening IDVs' level of expertise and capacity to lead gender equality training, as well as their cultural adaptability and sensitivity. The expertise that IDVs can bring to partner organizations for more effective and transformative gender mainstreaming impacts includes additional support for administrative, financial, human resources management.

Other support that IDVs could provide includes help with changing organizational procedures, facilitating the creation of organizational gender equality policies, and supporting programs and training to create changes in staff attitudes and behaviors (Tiessen 2007). Additional support that IDVs can bring to partner organization staff includes great attention to gender equality strategies beyond counting the number of women and men participants. This might involve researching the gender-specific needs of participants (such as childcare or travel-related needs that are impacted by the gender division of labor within the household). Monitoring and evaluation strategies can be enhanced through IDV support by delving deeper into gender relations and the underlying reasons for women's lack of participation in programs or the barriers to women's success in projects.

\section{Conclusion}

This paper examines some of the challenges of mainstreaming gender equality in partner organizations, with examples of resistance, externalizing gender problems as specific to 'beneficiary communities' rather than the organizations themselves, and, in some instances, the limited impact IDVs have on promoting gender-sensitive programming. Several challenges to gender mainstreaming were provided by partner organizations interviewed for this study.

Addressing these challenges requires strategic efforts to work with partner organization staff to uncover gender inequality perpetuating within and by the organization. Doing so will facilitate a more comprehensive approach to gender mainstreaming that focuses on the organizational practices, policies, procedures, monitoring, evaluating, and reporting on gender-related work. 
Opportunities for development organizations to look inward to their practices and organizational cultures can help partner organization staff reflect on the subtle and overt ways gender inequality is reproduced in day-to-day operation and acts of discrimination. They can also reveal the attitudes and behaviors that prevent changes needed to support gender equality. Uncovering these barriers within the organization is an important first step to understanding the obstacles to promoting gender equality in the communities where projects are implemented. Exposing the resistance to discussions of gender equality within partner organizations is a first step to recognizing the spaces where gender equality programs and interventions are needed. IDVs can play a valuable role in helping partner organization staff uncover some of the resistances to considering gender equality in the organization's day-today operations. IDVs bring fresh insights and outside perspectives that are valued by partner organization staff. These perspectives can be central to starting conversations about institutionalized gender inequalities within the organizations.

Throughout the interviews, attention to diverse forms of resistance to gender equality programming uncovered perceptions among some participants that gender equality programming favors women and can be seen to leave men and boys behind. Thus, addressing gender relations is central to effective gender equality programming, and IDVs can play an important role in the training and information sharing that explains gender inequality effectively to prevent misunderstandings.

The findings presented in this paper also revealed that gender inequality is generally observed as a problem to be tackled elsewhere and often, according to the partner organization staff, in the rural communities where projects are implemented. Recognizing gender inequality in these communities is a valuable starting point for considering some of the causes and consequences of discrimination or oppression of marginalized groups. As the participants noted, it is insufficient to view women as a cohesive group with the same experiences or challenges. Throughout the interviews, participants highlighted specific examples of intersecting factors that contributed to power imbalances, stigma, or diverse forms of disadvantage among women with specific lived experiences (specifically women who become widows, who are low income, or who have an excessive workload that prevents them from actively participating in project activities).

IDVs were generally considered valuable contributors to the organizations as they provide an essential role in supporting organizations in ways that allow them to integrate gender equality into a range of policies and practices that helped the organizations improve sex and gender-disaggregated data, enhanced training and capacity building, and improved policy and procedural approaches. IDVs also played an essential role in promoting gender equality and women's empowerment by providing new perspectives on gender issues and strategies to address gender inequality across various programs. Ensuring that IDVs come with gender equality 
expertise is crucial for supporting partner organization staff identified as priorities for improving their gender mainstreaming efforts. IDVs can therefore be an important contributor to gender mainstreaming within the organization and in the organization's programs. In order for IDVs to be more effective, the partner organizations highlighted the need for IDVs to come with a clear understanding of gender mainstreaming tools and strategies and knowledge of gender equality programming.

\section{Bibliography}

Cornwall, Andrea (2003): Whose voices? Whose choices? Reflections on gender and participatory development. In: World Development, vol. 31, no. 8, pp. 1325-1342.

Derbyshire, Helen (2002): Gender Manual. A practical guide for development policymakers and practitioners. London, https://www.rcrc-resilience-southeastasia.org/wp-content/ uploads/2015/12/gendermanual.pdf (28.10.2021).

EIGE (2016): institutional transformation. Gender Mainstreaming Toolkit. https://eige. europa.eu/gender-mainstreaming/toolkits/gender-institutional-transformation (23.6.2021).

Flood, Michael; Dragiewicz, Molly; Pease, Bob (2020): Resistance and backlash to gender equality. In: Aust J Soc Issues, pp. 1-16.

Hay, Katherine (2012): Engendering Policies and Programmes through Feminist Evaluation. Opportunities and Insights. In: Indian Journal of Gender Studies, vol. 19, no. 2, pp. 321340.

Jahan, Rounaq (1995): The Ilusive Agenda. Mainstreaming Women in Development. London.

Mannel, Jeneviève (2010): Gender mainstreaming Practice. Considerations for HIV/AID community organizations. In: AIDS care, vol. 22, no. 2, pp. 1613-1619.

Moser, Caroline; Moser, Annalise (2005): Gender Mainstreaming since Beijing. A review of success and limitations in international institutions. In: Gender and Development, vol. 13, no. 2, pp. 11-22.

Nelson, Diane M. (2015): Who Counts? the Mathematics of Death and Life after Genocide. Durham.

Parpart, Jane L. (2014): Exploring the transformative potential of gender mainstreaming in international development institutions. In: Journal of International Development, vol. 26, no. 3, pp. 382-395.

Radcliffe, Sarah A. (2015): Development Alternatives. In: Development and Change, vol. 46, no. 4 , pp. $855-874$.

Rao, Aruna; Sandler, Joanne; Kelleher, David; Miller, Carol (2016): Gender at work. Theory and practice for $21^{\text {st }}$ Century Organizations. Abington.

Tiessen, Rebecca (2007): Everywhere/Nowhere. Gender mainstreaming in development agencies. Bloomfield.

Tiessen, Rebecca (2019): What's New About Canada's Feminist International Assistance Policy and Why' More of the Same' Matters. https://journalhosting.ucalgary.ca/index.php/ sppp/article/view/MD\%20-\%2015 (4.6.2021). 
Tiessen, Rebecca; Rao, Sheila; Lough, Benjamin J. (2020): International Development Volunteering as Transformational Feminist Practice for Gender equality. In: Journal of Developing Societies, vol. 37, no. 1, pp. 30-56.

UN Women (N.D.): Gender Mainstreaming. www.unwomen.org/-/media/headquarters/ attachments/sections/library/publications/2020/gender-mainstreaming-strategy-forachieving-gender-equality-and-empowerment-of-women-girls-en.pdf?la=en\&vs=3849 (28.10.2021).

Valk, Minke (2000): An introduction: commitments to women and gender. In: Sarah, Cummings

Dam, Henk Van (ed.): Institutionalising gender equality: Commitment, policy, and Practice A Global Sourcebook (Gender, Society \& Development). Oxford.

Van Eerdewijk, Anouka; Davids, Tine (2014): Escaping the Mythical Beast. Gender Mainstreaming Reconceptualized. In: Journal of International Development, vol. 26, no. 3, pp. 303-316, DOI: 10.1002/jid.2947.

$\mathrm{Vu}, \mathrm{Vi}$ (2017): Why women should ditch Women's Day. Retrieved from VN Express. https://e. vnexpress.net/news/perspectives/why-women-should-ditch-women-s-day-3551008.html (17.10.2021).

Wallace, Tina (1998): Institutionalising gender in UK NGOs. In: Development in Practice, vol. 8, no, 2, pp. 159-172. 